\title{
Zidovudine treatment results in the selection of human immunodeficiency virus type 1 variants whose genotypes confer increasing levels of drug resistance
}

\author{
Paul Kellam, ${ }^{1 *}$ Charles A. B. Boucher, ${ }^{2}$ Jolanda M. G. H. Tijnagel ${ }^{2}$ and Brendan A. Larder ${ }^{1}$ \\ ${ }^{1}$ Antiviral Therapeutic Research Unit, Wellcome Research Laboratories, Langley Court, Beckenham, Kent BR3 3BS, \\ U.K. and "Antiviral Therapy Laboratory, Department of Virology, Academic Medical Centre, University of \\ Amsterdam, Meibergdreef 15, 1105 AZ Amsterdam, The Netherlands
}

High level resistance to $3^{\prime}$-azido-3'-deoxythymidine (AZT, zidovudine or Retrovir) is conferred by the presence of four or five mutations (Met-41 $\rightarrow$ Leu; Asp$67 \rightarrow$ Asn; Lys-70 $\rightarrow$ Arg; Thr-215 $\rightarrow$ Tyr or Phe; Lys$219 \rightarrow$ Gln) in the human immunodeficiency virus (HIV) reverse transcriptase. The order of appearance of these five mutations in asymptomatic patients during therapy has been studied. This has enabled us to propose a model for the acquisition of zidovudine resistance mutations during the treatment of high-risk asymptomatic HIV-infected individuals. A consistent acquisition pattern of mutations at codons 41,70 and 215 was observed in 17 individuals. Complex mixtures of HIV species containing different combinations of single and linked double resistance mutations were present early in zidovudine therapy in isolates from two patients studied in detail. From these mixtures the linked Leu-41/Tyr215 genotype outgrew all others initially. The development of each new virus population is likely to be mediated primarily by the increase in the level of drug resistance rather than changes in the growth kinetics of the virus. This leads us to conclude that one major driving force in the outgrowth of different mutant viruses is the selective advantage conferred by higher levels of drug resistance.

\section{Introduction}

Human immunodeficiency virus (HIV) isolates resistant to many compounds targeted to the virus-encoded enzyme reverse transcriptase (RT) have been identified during drug therapy or by selection in vitro (Fitzgibbon et al., 1992; Larder, 1992; Nunberg et al., 1991; Richman et al., 1991 a; St Clair et al., 1991). Variants of HIV highly resistant to zidovudine (AZT, Retrovir) have been isolated from AIDS patients receiving prolonged treatment (Boucher et al., 1990; Land et al., 1990; Larder et al., 1989; Richman et al., 1990; Rooke et al., 1989). High level resistance to zidovudine can be mediated by the acquisition of four specific amino acid substitutions in the RT of HIV-1 (Asp-67 $\rightarrow$ Asn; Lys-70 $\rightarrow$ Arg; Thr$215 \rightarrow$ Tyr or Phe; Lys-219 $\rightarrow$ Gln) (Larder \& Kemp, 1989). The relative sensitivities of certain combinations of these mutations have been reported (Larder et al., 1991). The appearance of the four amino acid substitutions has been studied in an asymptomatic patient population (Boucher et al., 1992a); this revealed an ordered but complex pattern of mutation acquisition. The substitution of Arg-70 $\rightarrow$ Lys is commonly detected first during zidovudine treatment. This mutation is only transient, appearing early during drug treatment and then disappearing as the mutation at codon 215 appears. The Lys-70 mutation then reappears, and finally mutations at codons 67 and 219 may develop with continuing treatment. A similar pattern of mutation acquisition was observed in a larger cohort of HIV-infected individuals at various stages of disease (Richman et al., 1991b). More recently a fifth mutation of Met to Leu at codon 41 has been shown to contribute to the development of zidovudine resistance (Kellam et al., 1992). Analysis of an asymptomatic patient population revealed that the mutation at codon 41 was present in 12 of 16 patients after 76 weeks of drug therapy (Kellam et al., 1992).

These studies indicate that complex interactions of mutations in RT are taking place in the virus population, involving gross changes in genotype and phenotype during the course of treatment. The occurrence of rapid sequence changes in the envelope glycoprotein of HIV-1 consisting of regular replacement by a succession of distinct viral populations has been demonstrated (Simmonds et al., 1991). Similar sequence variations have been observed in other HIV gene sequences (Delassus et 
al., 1991; Meyerhans et al., 1989; Pedroza Martins et al., 1991; Shen Pang et al., 1991). This has led to the view that HIV pathogenesis involves the rapid evolution of virus strains (Goodenow et al., 1989; Simmonds et al., 1991).

Here we report the detailed genotypic analysis of virus from infected peripheral blood lymphocytes (PBLs) from 17 initially asymptomatic HIV-positive individuals at all five codons associated with zidovudine resistance. Further, we report an extended genetic analysis of virus isolates from two patients from this cohort. A DNA sequence study of the RT-coding region from sequential isolates from these two patients revealed the appearance and turnover of various genotypes during treatment, resulting in the selection of variants with increasing resistance to zidovudine. Construction of a large panel of site-directed mutant viruses was undertaken to provide defined drug sensitivities of the genotypes observed, and to assess the in vitro growth properties of different mutants. The results presented enable us to propose a model for the acquisition of the five mutations that confer resistance to zidovudine.

\section{Methods}

Patient cohort. The Amsterdam asymptomatic cohort studied consisted of 18 high-risk (CD4<500) HIV-infected individuals of whom 17 were studied (Boucher et al., 1992a). From this cohort two individuals, Patient 199 (Pt199) and Patient 105 (Pt105), for whom multiple, sequential, cryopreserved PBL samples were available were chosen for detailed genetic analysis of viral zidovudine resistance.

Detection of Met-41 $\rightarrow$ Leu mutation by PCR. Selective PCR, used to discriminate wild-type (Met) from mutant (Leu) at codon 41 in cocultured PBLs, was performed essentially as described previously (Kellam et al., 1992) with the exception that different diagnostic oligonucleotides were used. To identify wild-type codon 41 , the primer pair used was BR (5' GGT GAT CCT TTC CAT CC $\left.3^{\prime}\right)$ with primer 5WR (5' TA CTA GAA ATT TGT ACA GAC A 3'). To identify mutant codon 41, primer BR was paired with either 5MR ( $5{ }^{\prime}$ TA CTA GAA ATT TGT ACA GAC T 3') to detect a TTG codon 41 , or $5 \mathrm{MCR}$ (5' TA CTA GAA ATT TGT ACA GAC C 3') to detect a CTG codon 41. The PCR reaction mixture $(100 \mu \mathrm{l})$ consisted of $25 \mathrm{~mm}-\mathrm{KCl}$, $1.5 \mathrm{~mm}-\mathrm{MgCl}_{\mathrm{g}}, 50 \mathrm{~mm}-\mathrm{Tris}-\mathrm{HCl} \mathrm{pH} 8.3,0.1 \mathrm{mg} / \mathrm{ml} \mathrm{BSA}, 0.2 \mathrm{~mm}$ of each of dATP, dCTP, dGTP and dTTP, $0.25 \mu \mathrm{g}$ of each primer and $2 \cdot 5$ units Taq polymerase (Cetus). The reactions were subjected to 30 cycles of $1 \mathrm{~min}$ at $94^{\circ} \mathrm{C}, 30 \mathrm{~s}$ at $50^{\circ} \mathrm{C}$ and $30 \mathrm{~s}$ at $72{ }^{\circ} \mathrm{C}$. This resulted in a PCR product of approximately 330 base pairs. The PCR products were separated through Tris-borate composite gels of $3 \%$ Nusieve $/ 1 \%$ agarose. The PCR was scored as described previously (Boucher et al., 1990).

Sequence analysis of PBL DNA. DNA was extracted from about $2 \times 10^{6}$ co-cultivated PBLs and subject to a nested PCR to amplify the full-length RT gene. The first PCR used primers RTOUT5 (5' GGA AAC CAA AAA TGA TAG GGG GAA TTG GAG G $3^{\prime}$ ) and RTOUT3 (5' TCT ACT TGT CCA TGC ATG GCT TC $3^{\prime}$ ). The PCR reaction mix $(100 \mu \mathrm{l})$ contained $25 \mathrm{~mm}-\mathrm{KCl}, 50 \mathrm{~mm}-\mathrm{Tris}-\mathrm{HCl} \mathrm{pH} 8 \cdot 3$, $\mathrm{l} .4 \mathrm{~mm}-\mathrm{MgCl}_{2}, 0.1 \mathrm{mg} / \mathrm{ml} \mathrm{BSA}, 0.2 \mathrm{~mm}$ of each of dATP, dCTP, dGTP, and dTTP, $100 \mathrm{ng}$ of each primer, $7.5 \%(\mathrm{v} / \mathrm{v})$ glycerol and 2.5 units Taq polymerase (Cetus). The PCR cycle consisted of 5 min at $94{ }^{\circ} \mathrm{C}$ followed by 35 cycles of 1 min at $94{ }^{\circ} \mathrm{C}, 2 \mathrm{~min}$ at $40^{\circ} \mathrm{C}, 4 \mathrm{~min}$ at $72^{\circ} \mathrm{C}$ and finishing with $10 \mathrm{~min}$ at $72^{\circ} \mathrm{C}$. The second PCR involved primers 5RTA ( $5^{\prime}$ TTG CAC TTT GAA TTC TCC CAT TAG $\left.3^{\prime}\right)$ and SRTB (5' TG TAC TTT GAA TTC CCC CAT TAG $3^{\prime}$ ) paired with $3 R T$ ( $5^{\prime}$ CTT ATC TAT TCC ATC TAG AAA TAG T $\left.3^{\prime}\right)$ and used $1.3 \mathrm{mM}-\mathrm{MgCl}_{2}$ in an otherwise unaltered PCR reaction mix. Ten $\mu \mathrm{l}$ of the first PCR reaction was re-amplified under the same conditions except that the extension time at $72{ }^{\circ} \mathrm{C}$ was 2 min rather than 4 min. This resulted in a full-length RT sequence with EcoRI and $X b a \mathrm{I}$ restriction enzyme sites introduced $5^{\prime}$ and $3^{\prime}$ respectively. The RT sequences were cloned using these sites into the vector pSP73. RT genecontaining clones were sequenced according to the protocol of Schuurman \& Keulen (1990) using Sequenase 2.0. Primers RT9 (5' AGT ACT GTT ACT GAT TT $\left.3^{\prime}\right)$ and Sp6 (5' GAT TTA GGT GAC ACT ATA G $3^{\prime}$ ) were used to sequence base pairs 90 to 255 containing the zidovudine resistance codons 41,67 and 70 . Primers RT11 (5'TAT GTA GGA TCT GAC TTA G 3') and RT6 (5' TTC TGT TAG TGC TTT GG 3') were used for sequencing of base pairs 600 to 825 containing the resistance codons 215 and 219 .

Cells and virus. The human T lymphoblastoid cell line C8166 (Salahuddin et al., 1983) was used to propagate HIV and for electroporation experiments. These cells were routinely maintained in RPMI 1640 medium supplemented with $10 \%(\mathrm{v} / \mathrm{v})$ fetal calf serum, plus antibiotics (RPMI 10). HT4LacZ-1 cells (Rocancourt et at., 1990) were used to determine the sensitivity of HIV to zidovudine. This cell line was maintained in Dulbecco's modified Eagle's medium supplemented with $10 \%$ fetal calf serum, plus antibiotics. MT-2 cells (Harada et al. 1985) were co-cultivated directly with patients' cryopreserved PBLs in RPMI 10 to obtain HIV stocks for sensitivity testing as described (Larder et al., 1989). Patient PBLs were also co-cultivated with donor (HIV seronegative) PBLs and DNA was extracted for PCR and DNA sequence analysis (Larder et al, 1991). To produce sitedirected mutant virus stocks, C8166 cells were co-transfected with a mixture of the RT-deleted proviral clone pHIVDRTBstEII and a functional mutant RT coding sequence derived from M13 clones as described (Kellam et al., 1992; St Clair et al,, 1991). Cell-free virus supernatants were harvested 12 to 14 days post-transfection and stored at $-70^{\circ} \mathrm{C}$.

Construction of mutant viruses. Mutants containing various combinations of the five zidovudine resistance mutations were created by sitedirected mutagenesis (Zoller \& Smith, 1984) of the previously described M13 RT clones HXB-2D. RTMF, RTMC/F and RTMC (Larder et al., 1991). All mutants were verified by nucleotide sequence analysis (Sanger et al., 1977). M13 replicative form DNA was prepared, and the mutant RT-coding regions were transferred into the HXB-2D genetic background by homologous recombination as described above.

Zidovudine sensitivity assay. Plaque-reduction assays were performed by infection of HT4LacZ-1 cell monolayers as described (Chesebro \& Wehrly, 1988; Larder et al. 1990). The 50\% inhibitory concentration $\left(\mathrm{IC}_{50}\right)$ values were derived from plots of percentage inhibition against zidovudine concentration.

Analysis of virus growth properties. C8166 cells $\left(4 \times 10^{4}\right)$ were infected with $4 \times 10^{3}$ p.f.u. $/ \mathrm{ml}$ of each mutant virus (Table 2 ). Six infections were set up per mutant. Virus was allowed to adsorb at $37^{\circ} \mathrm{C}$ for $1 \mathrm{~h}$. All six infections were then pooled, and the cells were pelleted and washed twice with $1 \mathrm{ml}$ of PBS. The cells were then resuspended in $6 \mathrm{ml}$ of RPMI 10 , and $1 \mathrm{ml}$ aliquots were plated out in 24-well tissue culture dishes. Samples were taken at time 0 (immediately after plating), 24, 48, 62,70 and $88 \mathrm{~h}$. At each time point a $1 \mathrm{ml}$ aliquot was removed from each well and the cell-free supernatant was stored at $-70^{\circ} \mathrm{C}$. After all samples had been taken the amount of infectious virus produced at each time point was determined by titrating the supernatants on 
HT4LacZ-1 monolayers. As controls, the growth properties of the clinical isolate PO26A (Larder et al., 1989) and of wild-type HXB-2D were determined.

\section{Results}

Acquisition of the five mutations conferring resistance to zidovudine

Seventeen initially asymptomatic HIV-positive individuals have been studied by selective PCR during the course of zidovudine therapy for the appearance of specific mutations at the RT gene codons $67,70,215$ and 219 associated with zidovudine resistance (Boucher $e t$ al., 1992a). This study was further extended by the analysis of codon 41 in these patients' PBL populations. All cocultured PBL samples obtained before treatment were wild-type at codons 41,70 and 215. In addition, for three patients analysed, codons 67 and 219 were also wild-type before treatment (Table 1). A general pattern of resistance mutation acquisition could be observed. In eight of 17 patients (patients 385 to 276, Table 1) the first mutation to appear was at codon 70 either as a mutant population or a mixed population of wild-type and mutant sequences (Boucher et al., 1992a). However, with continued treatment this mutation returned to a wildtype or mixed population. As this occurred the mutation at codon 215 appeared, closely followed by the acquisition of the mutation at codon 41 . Both mutations remained in the PBL population once present. In five of 17 patients (patients 105 to 346 ) a similar pattern was observed with the acquisition of mutations at codons 41 and 215 . By 76 to 132 weeks of zidovudine therapy 13 of $17(76 \%)$ patients harboured HIV with mutations at codons 41 and 215 . It is likely that for patients 105 to 346 the transient appearance of a mutation at codon 70 occurred between the times of sampling. This was indeed the case for patient 105 who acquired a mutant codon 70 population between 12 and 24 weeks (Fig. 2). For patient 181, a mixed population of wild-type and mutant sequences at codons 70 and 215 arose by 28 weeks and these became mutant at codon 215 alone by 96 weeks. For patient 232 a mutation at codon 215 was observed only after 112 weeks of drug therapy, all other codons remaining wild-type. For patients 56 and 1123 populations with a mutation at codon 70 only were observed.

\section{Detailed analysis of two patients}

The temporal appearance of mutations associated with zidovudine resistance in individual patients was indicated from the analysis of 17 asymptomatic HIV-positive individuals. In order to determine more accurately the exact order of appearance of the five resistance mutations, isolates from two patients previously described,
Table 1. Longitudinal analysis of $R T$ genes during treatment of $17 \mathrm{HIV}$-positive individuals with zidovudine $(A Z T)$

\begin{tabular}{|c|c|c|c|c|c|c|}
\hline \multirow{2}{*}{$\begin{array}{l}\text { Patient } \\
\text { code* }^{*}\end{array}$} & \multirow{2}{*}{$\begin{array}{l}\text { Duration } \\
\text { of AZT } \\
\text { therapy } \\
\text { (weeks) }\end{array}$} & \multicolumn{5}{|c|}{ Reverse transcriptase codon $\dagger$} \\
\hline & & 41 & 67 & 70 & 215 & 219 \\
\hline \multirow[t]{3}{*}{385} & 0 & W & - & W & W & - \\
\hline & 24 & W & - & $\mathbf{M}$ & W & - \\
\hline & 96 & M & W & $\mathrm{X}$ & M & W \\
\hline \multirow[t]{3}{*}{316} & 0 & W & - & W & W & - \\
\hline & 24 & W & - & $\mathbf{M}$ & $\mathrm{X}$ & - \\
\hline & 98 & $\mathbf{M}$ & W & W & $\mathbf{M}$ & W \\
\hline \multirow[t]{3}{*}{278} & 0 & W & - & W & W & - \\
\hline & 32 & W & - & $\mathrm{x}$ & $x$ & - \\
\hline & 96 & M & W & W & M & W \\
\hline \multirow[t]{3}{*}{734} & 0 & W & - & W & W & - \\
\hline & 20 & W & - & $\mathrm{x}$ & W & - \\
\hline & 76 & $X$ & W & $\mathrm{X}$ & M & W \\
\hline \multirow[t]{2}{*}{199} & 0 & W & W & W & W & W \\
\hline & 12 & W & W & M & W & W \\
\hline AIDS, 104 weeks & 132 & M & W & $\mathrm{X}$ & M & W \\
\hline \multirow{3}{*}{107} & 0 & W & - & W & W & - \\
\hline & 28 & W & - & M & W & - \\
\hline & 88 & M & W & W & $\mathbf{M}$ & W \\
\hline \multirow[t]{2}{*}{373} & 0 & W & W & W & $W$ & W \\
\hline & 28 & W & W & M & W & W \\
\hline AIDS, 117 weeks & 109 & $\mathrm{M}^{*}$ & W & W & M & W \\
\hline \multirow[t]{3}{*}{276} & 0 & W & - & W & W & - \\
\hline & 28 & W & - & $\mathrm{X}$ & W & - \\
\hline & 100 & $\mathbf{M}^{*}$ & W & W & M & W \\
\hline \multirow[t]{2}{*}{105} & 0 & W & W & W & w & W \\
\hline & 24 & $\mathrm{X}$ & - & W & $\mathbf{M}$ & W \\
\hline AIDS, 80 weeks & 86 & M & $\mathrm{X}$ & $\mathrm{X}$ & M & W \\
\hline \multirow[t]{2}{*}{393} & 0 & W & - & W & W & - \\
\hline & 28 & $M^{*}$ & - & W & $x$ & - \\
\hline AIDS, 82 weeks & 103 & $\mathrm{M}^{*}$ & W & W & M & W \\
\hline \multirow[t]{3}{*}{182} & 0 & W & - & W & W & - \\
\hline & 28 & - & - & W & $\mathbf{M}$ & - \\
\hline & 100 & $\mathbf{M}$ & W & W & $\mathbf{M}$ & $W$ \\
\hline \multirow[t]{3}{*}{308} & -2 & W & - & W & W & - \\
\hline & 32 & W & - & W & W & - \\
\hline & 96 & M & W & W & $M$ & W \\
\hline \multirow[t]{3}{*}{346} & 0 & W & - & W & W & - \\
\hline & 28 & W & - & W & $\mathrm{M}$ & - \\
\hline & 108 & $\mathbf{M}$ & W & W & $\mathbf{M}$ & W \\
\hline \multirow[t]{3}{*}{181} & 0 & W & - & W & W & - \\
\hline & 28 & W & - & $\mathrm{X}$ & $X$ & - \\
\hline & 96 & W & W & W & $\mathrm{M}$ & W \\
\hline \multirow[t]{3}{*}{56} & 0 & W & - & W & W & - \\
\hline & 24 & W & - & $\mathbf{M}$ & W & - \\
\hline & 96 & W & W & $X$ & W & W \\
\hline \multirow[t]{3}{*}{232} & 0 & W & - & W & W & - \\
\hline & 28 & W & - & W & W & - \\
\hline & 112 & W & W & W & $\mathbf{M}$ & W \\
\hline 1123 & 0 & W & - & W & W & - \\
\hline & 28 & W & - & W & W & - \\
\hline & 88 & W & W & M & W & W \\
\hline
\end{tabular}

* For patients who progressed to AIDS during treatment, the time AIDS developed is indicated.

$\dagger \mathrm{W}$, wild-type codon; $\mathrm{X}$, mixture of wild-type and mutant codon; $M$, mutant codon; $M^{*}$, alternative $C T G$ codon for the mutation at codon 41 .

Pt199 and Pt105 (Boucher et al., 1992a), for whom multiple longitudinal samples were available during zidovudine therapy, were analysed by selective PCR. 


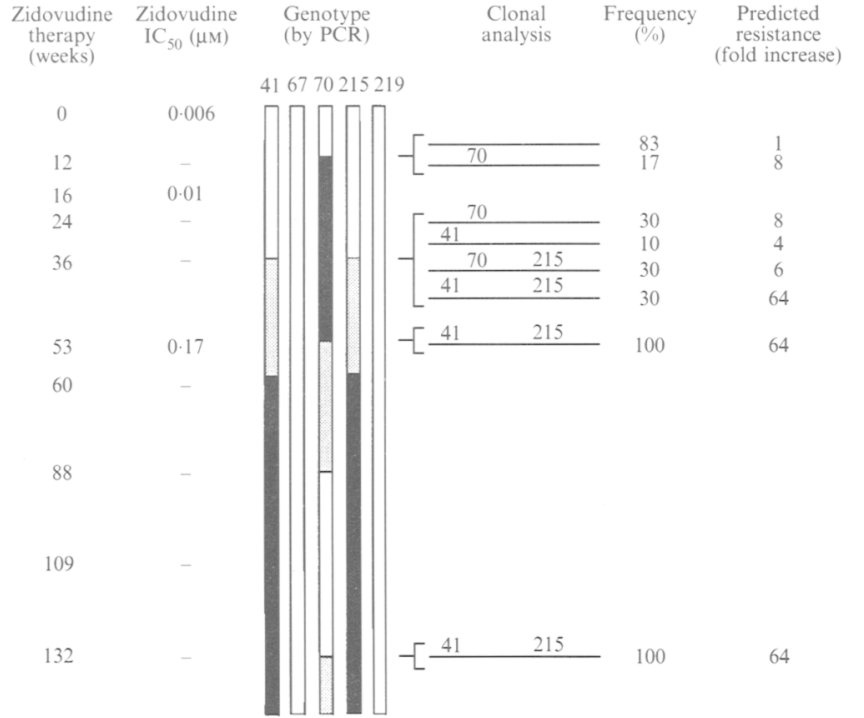

Fig. 1. Genotype and drug sensitivity analysis of multiple sequential samples from Pt199. Zidovudine sensitivities were assessed in the HeLa $\mathrm{CD}^{+}$plaque reduction assay. Mutations associated with zidovudine resistance were monitored by selective PCR. Mutations are shown by shadings of bars: [] , wild-type detected; $[$, both wild-type and mutant detected; $\boldsymbol{\square}$, mutant only detected. The linkage patterns of resistance mutations were determined by sequence analysis of cloned RT coding sequences. The frequency and predicted increase in drug resistance above wild-type is shown for each genotype identified.

\section{(i) Patient 199}

A homogeneous population with a mutation at codon 70 was detected by PCR at 12 weeks of therapy (Fig. 1). Subsequently a complex population of mutant codon 70 and mixtures of wild-type and mutants at codons 41 and 215 arose after 36 weeks of therapy. This was followed by the development of mixtures of mutants and wild-type at all three codons by 53 weeks. By 60 weeks of treatment, homogeneous populations were present with mutations at codons 41 and 215, and at codon 70 a mixed population of wild-type and mutant variants was still present. The mutation at codon 70 had disappeared completely after 88 weeks of therapy, and subsequently reappeared as part of a mixed population of mutants and wild-type by 132 weeks.

\section{(ii) Patient 105}

The first mutation detected was at codon 70 after 12 weeks of therapy and was present as a mixed population of wild-type and mutant sequences which became completely wild-type at codon 70 as mutations at codons 41 and 215 occurred by 24 weeks (Fig. 2). By 36 weeks a homogeneous population of mutants at codons 41 and 215 had developed. Also at this time the mutation at codon 70 reappeared in a mixed population of mutant and wild-type. This mixture at codon 70 persisted until 136 weeks of treatment when the population became homogeneously mutant. In Pt105 the mutation at codon

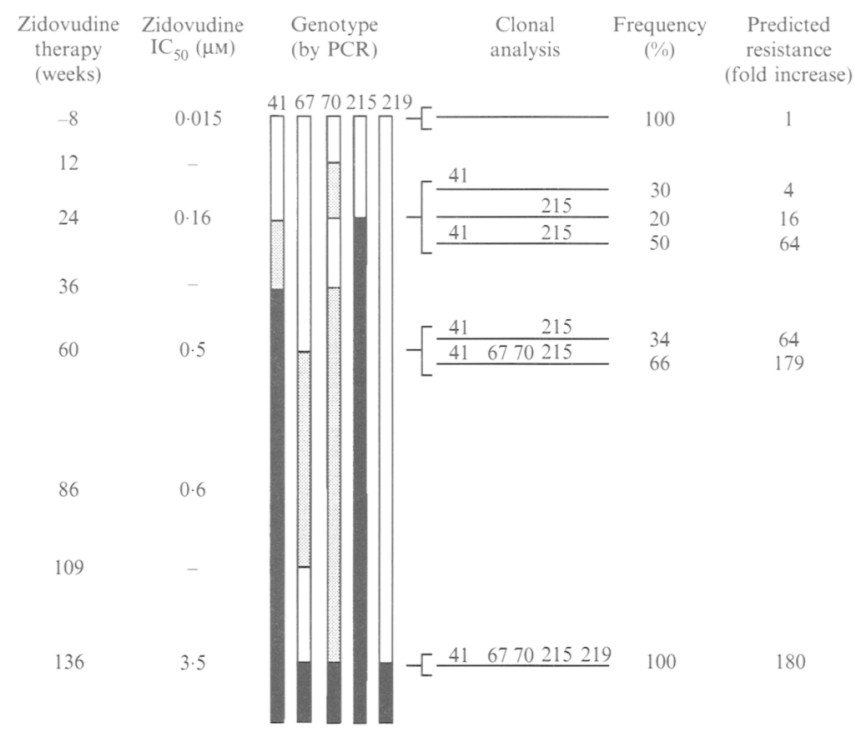

Fig. 2. Genotype and drug sensitivity analysis of multiple sequential samples from Pt105. Zidovudine sensitivities were assessed in the HeLa $\mathrm{CD}^{+}$plaque reduction assay. Mutations associated with zidovudine resistance were monitored by selective PCR. Mutations are shown by shadings of bars: $\square$, wild-type detected; $⿴ 囗 ⿱ 一 一)$, both wild-type and mutant detected; $\boldsymbol{\square}$, mutant only detected. The linkage patterns of resistance mutations were determined by sequence analysis of cloned RT coding sequences. The frequency and predicted increase in drug resistance above wild-type is shown for each genotype identified.

67 developed, first as a mixture between 60 and 109 weeks and then subsequently returned to wild-type. The mutation then reappeared as a homogeneous mutant population along with the mutation at codon 219 . This resulted in Pt105 having mutations at all five resistance codons at 136 weeks.

It is interesting to note that in both patients the sequence of mutation appearance was very similar but the timing of the appearance of mutations was quite distinct, with Pt105 exhibiting a faster rate of mutation acquisition. At all times of sampling the patients harboured syncytium-inducing isolates of HIV as shown by their ability to replicate in the T cell line MT-2 (Boucher et al., 1992a). Virus stocks prepared were assayed for sensitivity to zidovudine (Fig. 1 and 2). The level of drug resistance increased with continuing drug therapy resulting in the acquisition of a virus with a highly resistant phenotype in Pt105 by 136 weeks.

\section{Clonal sequence analysis of mutation acquisition}

PCR analysis revealed the appearance of complex mixtures of wild-type and mutant sequences at various times of drug treatment. From these mixtures changes in the overall genotype of the population arose. Therefore, we wished to determine whether there were linkage 

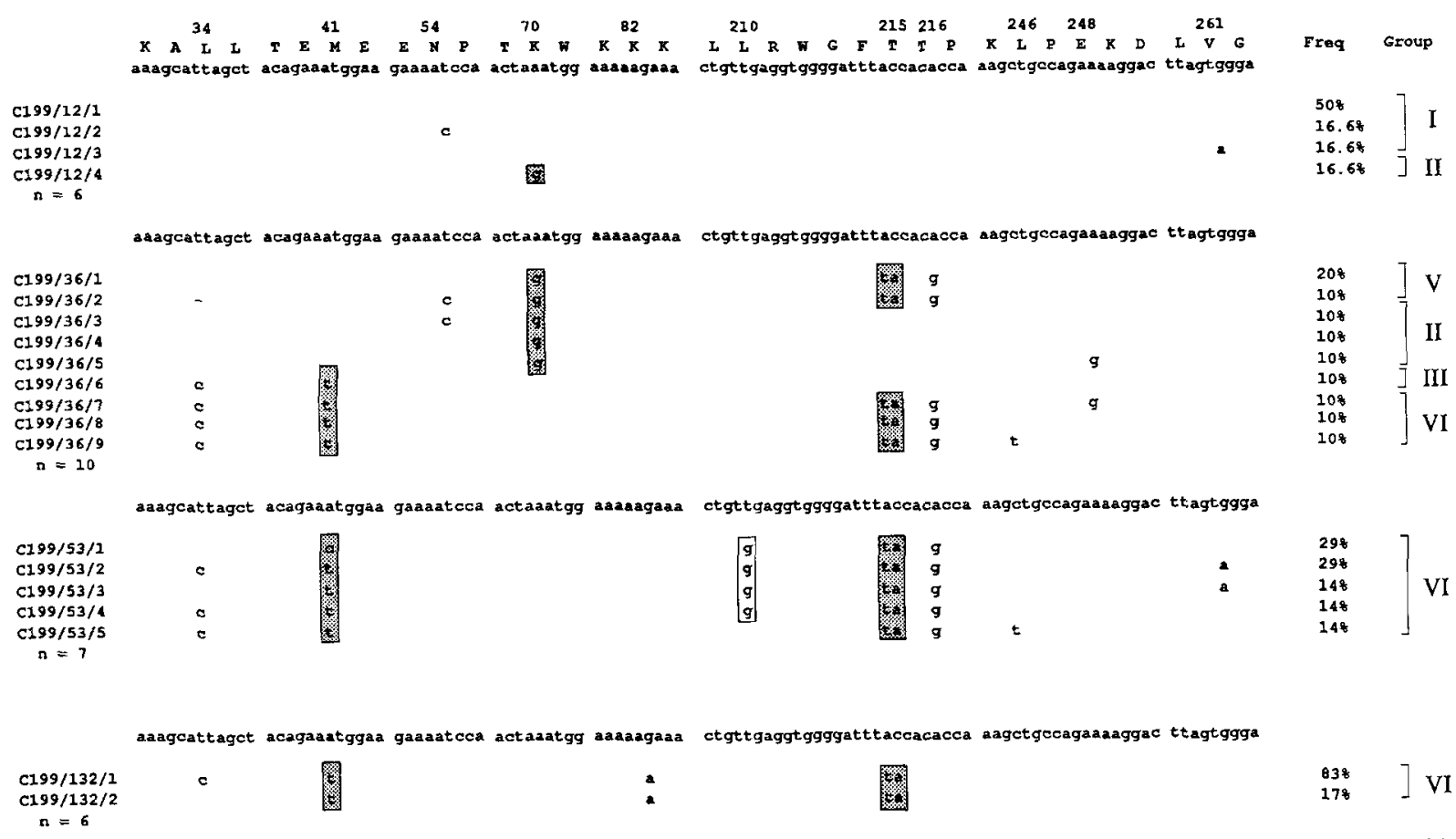

Fig. 3. Regions of RT coding sequence for multiple clones from Pt 199 at 0, 36, 53 and 132 weeks of zidovudine treatment. Nucleotide substitutions differing from the predominant time 0 sequence (shown in full) are indicated. The frequency of a given sequence type is shown on the right and the number of clones $(n)$ sequenced at each time is indicated. The grouping of sequences was based on the identified zidovudine resistance genotype, Group I containing wild-type sequences, Group II containing Arg-70 sequences, Group III containing Leu-41 sequences, Group IV containing Tyr-215 sequences, Group V containing linked Arg-70 and Tyr- 215 sequences, and Group VI containing linked Leu-41 and Tyr-215 sequences. Nucleotide substitutions conferring amino acid changes are indicated [] , with zidovudine resistance mutations being shown as 圆.

patterns of the five mutations in the changing populations. To address this, DNA sequence analysis was performed on cloned RT sequences from Pt199 at 12, 36, 53 and 132 weeks, and Pt105 at -8, 24,60 and 136 weeks of drug therapy. The predominant wild-type strain detected at the start of drug therapy was taken as the reference sequence when making sequence comparisons. The frequency of the appearance of each population is shown in Fig. 3 and Fig. 4 for patients 199 and 105 respectively, with sequences grouped with respect to their zidovudine resistance genotype.

(i) Patient 199

At 12 weeks of treatment a mixed population of predominantly wild-type sequences (Group I) of different populations was observed, along with the presence of a population of RT genes containing a mutation at codon 70 (Group II, C199/12/4). By 36 weeks, a complex mixture of mutant genotypes was seen. Ten clones were sequenced and populations consisting of unlinked amino acid substitutions at codons 41 (Group III, 1/10) and 70 (Group II, 3/10) were seen, as well as populations of RT genes with linked mutations at codons 41 and 215 (Group VI, 3/10), or 70 and 215 (Group V, 3/10). By 53 weeks of treatment the population had shifted to contain all linked 41 and 215 mutations (Group VI, 7/7), and this genotype persisted at 132 weeks. Interestingly, at 53 weeks two of seven variants sequenced contained the alternative CTG codon for leucine at amino acid 41 (C199/53/1) rather than the more frequently observed TTG codon. From all the sequence variation observed, amino acid substitutions resulted only at the zidovudine resistance codons and at codon 210 (Leu $\rightarrow$ Trp).

(ii) Patient 105

At 8 weeks before the start of drug therapy all strains sequenced were wild-type with respect to the known zidovudine resistance codons. Four clones sequenced at this time point revealed the presence of three different wild-type virus genotypes. By 24 weeks of treatment mutations at codons 41 and 215 were observed. In $50 \%$ (five) of the clones sequenced the mutations at codons 41 and 215 were linked on the same RT coding sequence, namely (Group VI) C105/24/1 and -2 . The remaining clones contained unlinked mutations at codons 41 (Group III, 3/10) or 215 (Group IV, 2/10). With continued drug therapy the appearance of mutations at codons 67 and 70 was observed at 60 weeks. At this time 


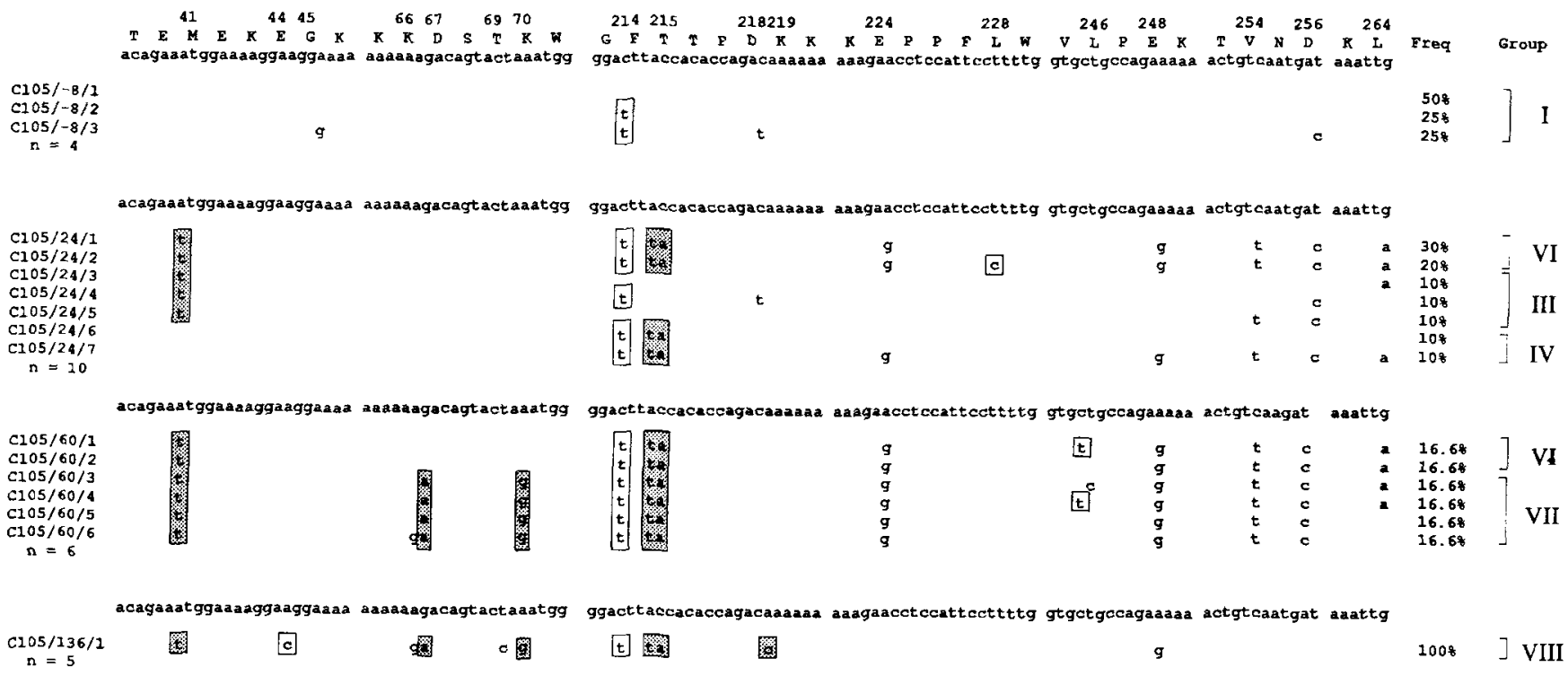

Fig. 4. Regions of RT coding sequence for multiple clones from Pt 105 at $-8,24,60$ and 136 weeks of zidovudine treatment. Nucleotide substitutions differing from the predominant time -8 sequence (shown in full) are indicated. The frequency of a given sequence type is shown on the right and the number of clones ( $n$ ) sequenced at each time is indicated. The grouping of sequences was based on the identified zidovudine resistance genotype, Group I containing wild-type sequences, Group II containing Arg-70 sequences, Group III containing Leu-41 sequences, Group IV containing Tyr-215 sequences, Group V containing linked Arg-70 and Tyr-215 sequences, Group VI containing linked Leu-41 and Tyr-215 sequences, Group VII containing linked Leu-41, Asn-67, Arg-70 and Tyr-215 sequences, and Group VIII containing sequences with all five zidovudine resistance mutations. Nucleotide substitutions conferring amino acid changes are indicated $\square$, with zidovudine resistance mutations being shown as 粼.

a population of linked 41 and 215 mutations (C105/60/1 and -2 ) remained but the predominant genotype at this time consisted of linked mutations at codons $41,67,70$ and 215 (Group VII). Interestingly, no linked mutations at codons 41,67 and 215 or 41,70 and 215 were seen. By 136 weeks a predominant sequence group containing all five resistance mutations was observed (Group VIII). From all of the sequence variation present, amino acid substitutions occurred only at the zidovudine resistance codons and at codons 44 (Glu $\rightarrow$ Asp), 214 (Phe $\rightarrow$ Leu), 228 (Leu $\rightarrow$ Pro) and 246 (Leu $\rightarrow$ Pro). These results with respect to the zidovudine resistance mutations are summarized in Fig. 1 and 2.

\section{Analysis of site-directed mutant viruses}

In order to understand why certain mutant virus populations outgrow others in the face of zidovudine therapy, a series of site-directed mutant viruses was constructed with different combinations of the five resistance mutations. Virus recovered was assayed for sensitivity to zidovudine (Table 2). The first resistance mutation detected was Lys-70 to Arg (HIVRTMJ). This had previously been shown to result in an increase in drug resistance of eightfold (Larder et al., 1990). Virus containing only a single amino acid substitution at codon 41 of Met to Leu (HIVRTML) has been shown to exhibit
Table 2. Zidovudine sensitivity of HIV variants with defined mutations in $R T$

\begin{tabular}{|c|c|c|c|c|c|c|c|}
\hline \multirow[b]{2}{*}{$\begin{array}{l}\text { Virus } \\
\text { mutant }\end{array}$} & \multicolumn{5}{|c|}{ Genotype } & \multirow{2}{*}{$\begin{array}{l}\text { Zidovudine } \\
\mathrm{IC}_{50}(\mu \mathrm{M})^{*}\end{array}$} & \multirow{2}{*}{$\begin{array}{l}\text { Zidovudine } \\
\text { resistance } \\
\text { (fold } \\
\text { increase) }\end{array}$} \\
\hline & $\begin{array}{l}41 \\
\mathbf{M}\end{array}$ & $\begin{array}{l}67 \\
\mathrm{D}\end{array}$ & $\begin{array}{l}70 \\
\mathrm{~K}\end{array}$ & $\begin{array}{c}215 \\
\mathrm{~T}\end{array}$ & $\begin{array}{c}219 \\
\mathrm{~K}\end{array}$ & & \\
\hline HXB-2 & & & & & & 0.01 & - \\
\hline HIVRTML & $\mathrm{L}$ & . & & & & 0.04 & 4 \\
\hline HIVRTMJ & & 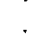 & $\mathbf{R}$ & 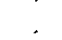 & 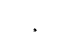 & 0.08 & 8 \\
\hline HIVRTMF & & 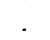 & . & $Y$ & 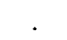 & 0.16 & 16 \\
\hline HIVRTMN & $\mathbf{L}$ & . & & $\mathrm{Y}$ & . & 0.64 & 64 \\
\hline HIVRTMW & $\mathrm{L}$ & . & $\mathbf{R}$ & & . & 0.09 & 9 \\
\hline HIVRTMO & & . & $\mathbf{R}$ & $\mathrm{Y}$ & . & 0.06 & 6 \\
\hline HIVRTMP & $\mathrm{L}$ & & $\mathrm{R}$ & $\bar{Y}$ & 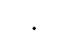 & 0.34 & 34 \\
\hline HIVRTMU & $\mathrm{L}$ & $\mathbf{N}$ & . & $\mathrm{Y}$ & & 0.43 & 43 \\
\hline HIVRTMC/F & & $\mathrm{N}$ & $\dot{\mathrm{R}}$ & $\mathrm{Y}$ & & 0.31 & 31 \\
\hline HIVRTMQ & $\mathrm{L}$ & $\mathrm{N}$ & $\mathbf{R}$ & $\mathrm{Y}$ & & 1.79 & 179 \\
\hline HIVRTMC & & $\mathrm{N}$ & $\mathrm{R}$ & $\mathrm{F}$ & $\mathrm{Q}$ & 1.47 & 147 \\
\hline HIVRTMR & $\mathrm{L}$ & $\mathrm{N}$ & $\mathbf{R}$ & $\mathrm{F}$ & $Q$ & 0.51 & 51 \\
\hline HIVRTMC/Y & 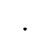 & $\mathrm{N}$ & $\mathbf{R}$ & $\mathrm{Y}$ & $\mathrm{Q}$ & $1 \cdot 21$ & 121 \\
\hline HIVRTMS & $\mathrm{L}$ & $\mathrm{N}$ & $\mathbf{R}$ & $\mathrm{Y}$ & $\mathrm{Q}$ & $1 \cdot 80$ & 180 \\
\hline
\end{tabular}

* Zidovudine sensitivity was assessed by plaque reduction assay. Mean $\mathrm{IC}_{50}$ values are shown, which were derived from between two and five independent sensitivity assays.

a fourfold increase in zidovudine resistance (Kellam et al., 1992). The double nucleotide substitution resulting in the amino acid change of Thr-215 to Tyr (HIVRTMF) resulted in a 16-fold increase in resistance (Larder et al., 
1990). Sequence analysis of Pt199 and Pt105 RT coding regions showed that these single mutations became linked relatively early in drug therapy to give rise to different double mutants. Site-directed mutant viruses containing these combinations revealed large differences in sensitivity to zidovudine. The combination of mutations at codons 41 and 215 (HIVRTMN) was shown to result in a greater than additive increase in resistance (64fold) compared with the mutations singly (Kellam et al., 1992). The combination of Arg-70 and Tyr-215 (HIVRTMO) resulted in a threefold decrease in the level of resistance relative to Tyr-215 alone. The combination of Leu-41 and Arg-70 (HIVRTMW) yielded a virus that was only ninefold more resistant to zidovudine than wild-type virus, but still less resistant than Tyr- 215 alone. HIVRTMP which has mutations at codons 41,70 and 215 resulted in a virus with a twofold decrease in resistance relative to HIVRTMN (41 and 215). This particular genotype was not seen in the sequencing of the clinical isolate RT genes. The site-directed mutant virus with mutations at codons 41, 67 and 215 (HIVRTMU) exhibited a sensitivity to zidovudine at a level similar to that of HIVRTMP (41, 70 and 215). However, this genotype was also not observed during the sequencing of the clinical isolates. The mutant virus containing amino acid substitutions at codons 41, 67, 70 and 215 (HIVRTMQ) has been shown to have a highly resistant phenotype (Kellam et al., 1992). Further addition of the mutation at codon 219 to this background (HIVRTMS; $41,67,70,215$ and 219) did not result in any appreciable increase in zidovudine resistance. Although both these combinations were seen during the sequence analysis of clinical isolates the significance of the HIVRTMS (41, $67,70,215,219)$ genotype and the mutation at codon 219 are unknown. The highly resistant viruses HIVRTMC $(67,70,215 \mathrm{~F}, 219)$ and HIVRTMC/Y $(67,70,215 \mathrm{Y}$, 219) differed only in the amino acid at codon 215 , HIVRTMC having Phe-215 and HIVRTMC/Y having Tyr-215. This did not result in any dramatic difference in sensitivity to zidovudine. However, addition of Leu-41 into HIVRTMC (HIVRTMR; 41, 67, 70, 215F, 219) resulted in a greater than threefold decrease in resistance. The presence of Leu-41 in HIVRTMC/Y (HIVRTMS; $41,67,70,215 \mathrm{Y}, 219)$ resulted in a small increase in resistance to an $\mathrm{IC}_{50}$ of $1.8 \mu \mathrm{M}$ relative to the sensitivity of HIVRTMC/Y $\left(\mathrm{IC}_{50} 1.21 \mu \mathrm{M}\right)$. The genotypes of HIVRTMC and HIVRTMC/Y were not seen in the sequence analysis of the asymptomatic cohort.

\section{Replication characteristics of the site-directed mutant viruses}

To determine whether the different RT genotypes could

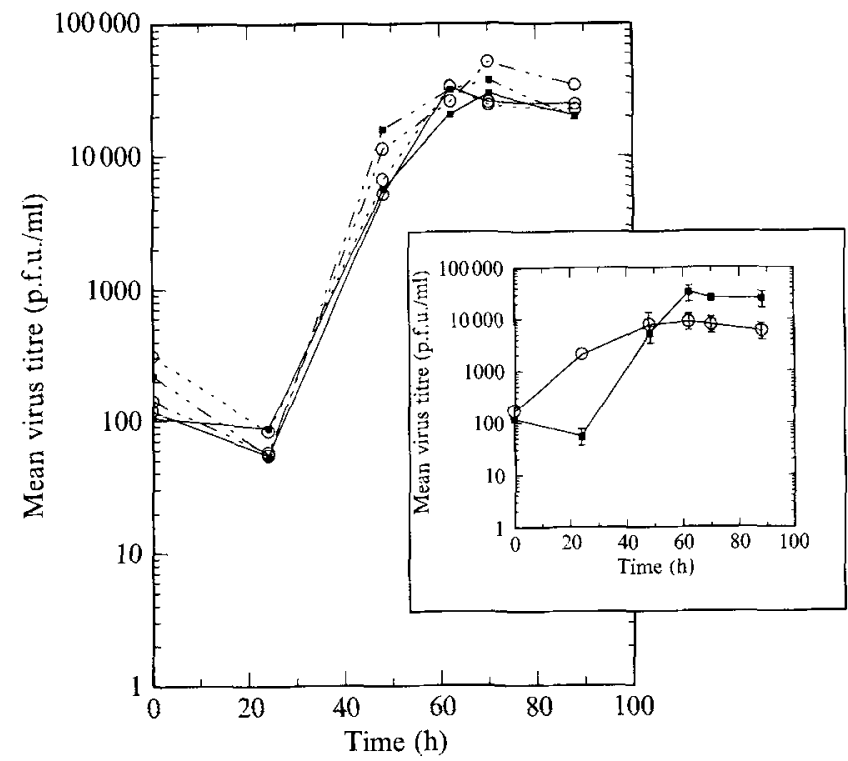

Fig. 5. Growth kinetics of four site-directed mutant viruses, HIVRTML

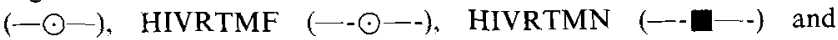
HIVRTMS (--- $\odot--)$, compared with the parental wild-type virus HXB-2D ( - - Growth analysis was performed between two and four times for each virus with the average virus titre at each time being plotted. Standard error bars are shown for HXB-2D (inset). The same degree of variation was observed with all assays. Differences in growth between $\mathrm{HXB}-2 \mathrm{D}$ and the clinical isolate $\mathrm{PO} 26 \mathrm{~A}(-\mathrm{O}-)$ are shown (inset).

contribute to the selective outgrowth of the mutant viruses, the growth properties of the site-directed mutant viruses HIVRTML, -J, -F, -N, -O, -P, -C/F, -Q, -C, - R, $-\mathrm{C} / \mathrm{Y}$ and $-\mathrm{S}$ (Table 2 ) were compared with wild-type. As all the mutations were present in the HXB-2D genetic background it was possible to study the contributions of the individual mutations to the growth of the virus, rather than growth differences attributable to other virus gene products. C8166 cells were infected at an m.o.i. of 0.1 and the production of viable virus was followed over $88 \mathrm{~h}$ (Fig. 5). Assays were performed between two and four times for each mutant. All mutant viruses produced the same general growth characteristics, very similar to wild-type HXB-2D. Virus production started at approximately $24 \mathrm{~h}$ post-infection (p.i.) and reached a peak at about $60 \mathrm{~h}$ p.i. The virus titre then started to decline after $70 \mathrm{~h}$. Any slight differences in the growth curves of the mutants could be accounted for by variation between assays. The errors associated with repeated assaying are shown in the inset (Fig. 5) for HXB-2D wild-type. The assay was able to detect gross differences in the growth of viruses, as shown by the different growth kinetics of the clinical isolate PO26A (Larder et al., 1989) (inset). The replication rate of $\mathrm{PO} 26 \mathrm{~A}$ differed from that of the other mutant viruses in that virus production was already 


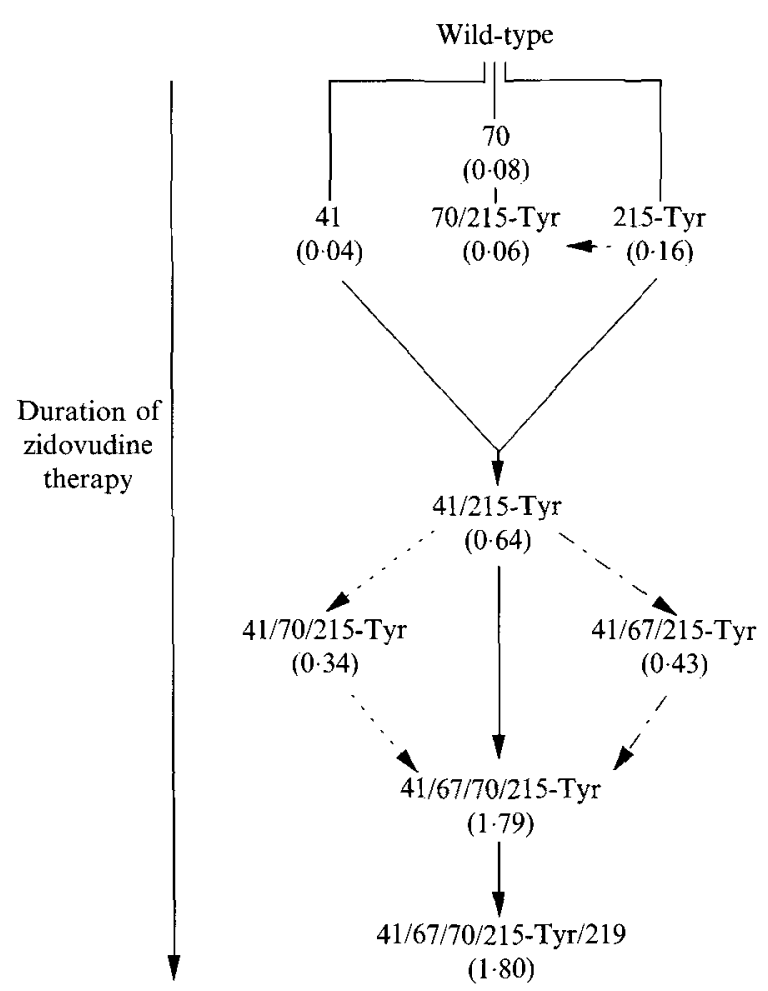

Fig. 6. Proposed model for sequential acquisition of the five mutations conferring resistance to zidovudine. Numbers represent a mutation at the codon indicated. The sensitivity to zidovudine $(\mu \mathrm{M})$ for each genotype is shown in parentheses.

significant at $20 \mathrm{~h}$ p.i., and peak virus production was reached at about $40 \mathrm{~h}$.

\section{Discussion}

Information gathered from this study and previous work on zidovudine resistance enables us to propose a model for the pattern of resistance mutation acquisition in highrisk asymptomatic HIV-infected individuals (Fig. 6). Changes in virus populations over time can be attributed to a change in the virus's ability to replicate in a given environment. This can be achieved by an increase in the replication rate of a virus in the absence of drug therapy, and/or a selective advantage conferred on drug-resistant virus in the presence of the drug. Growth analysis in the absence of zidovudine of site-directed mutant virus containing different combinations of zidovudine resistance mutations revealed no dramatic differences in their replication rates in the $T$ cell line C8166. Gross differences in the ability of HIV-1 strains to replicate could be shown in the assay system used, as demonstrated by differences between the clinical isolate PO26A and HXB2-D. Other cell lines were not studied and could possibly reveal differences in growth rates. Other studies have shown that wild-type virus has a very slight growth advantage over a Tyr-215 (HIVRTMF) mutant in MT2 cells. In these experiments wild-type virus was able to outgrow and become the dominant virus from an initially mixed population with Tyr-215 (HIVRTMF) after 20 passages in vitro (Boucher et al., 1993). Taken together, these results suggest that the mutant viruses replicate sufficiently similarly in vitro that no mutant would quickly outgrow another. Three groups have reported the gradual return to wild-type of resistant HIV after the cessation of zidovudine therapy (Albert et al., 1992; Boucher et al., 1993; Land et al., 1991). All showed that the change from mutant to wild-type isolates does not occur rapidly and may be dependent on the duration of therapy. This was suggested to indicate that the in vivo replicative capacities of resistant variants are similar to those of sensitive variants (Albert et al., 1992). Therefore, the growth rates conferred by resistance mutations in RT alone are unlikely to contribute dramatically to the change in virus populations during the development of drug resistance.

Each new virus population that becomes dominant does however have a significant increase in resistance to zidovudine, resulting in a selective growth advantage. The first mutation to appear, at codon 70 , confers the highest level of resistance (eightfold) of all the mutations due to a single nucleotide substitution (Larder et al., 1990; Kellam et al., 1992). During the initial stages of zidovudine resistance development additional populations of mutants with single amino acid changes co-exist, namely Leu-41 or Tyr-215, the latter conferring the highest level of resistance of all the single amino acid substitutions (16-fold). Different combinations of these mutations can become linked to give double mutants conferring varying levels of drug resistance. Some of these combinations were observed in the clinical samples studied, namely $70 / 215$ and $41 / 215$. The linked phenotype Leu-41/Tyr-215, conferring a greater than 60 -fold increase in resistance, outgrows all other mutants initially, and appears to be a major landmark during the development of zidovudine resistance. Of the isolates from two patients studied in detail all became homogeneously mutant at these codons between 36 and 60 weeks of therapy. Of a total of 17 patients studied (including Pt105 and Pt199) 13 had homogeneous populations of linked $41 / 215$ after 76 to 132 weeks of zidovudine treatment. The appearance of homogeneous populations of mutants at the remaining three codons occurred only in Pt105 after 136 weeks. The transition from the double mutant $41 / 215$ to the highly resistant variant $41 / 67$ / $70 / 215$ could proceed via the intermediates $41 / 70 / 215$ and/or $41 / 67 / 215$, although neither of these genotypes was seen during the sequence analysis. It is possible the $41 / 70 / 215$ and $41 / 67 / 215$ genotypes occur clinically, as other combinations of mutations that confer lower 
resistance levels were observed during the sequence analysis, for example $70 / 215$. The $41 / 70 / 215$ and $41 / 67 / 215$ genotypes may be able to replicate well in different cellular reservoirs. In these reservoirs the maximum zidovudine concentration may vary from the level attainable in PBLs (Perno et al., 1992). When these genotypes acquire further mutations and become highly resistant they would then have a selective advantage and outgrow the $41 / 215$ double mutant in the general population. It is important to remember, however, that the patients in this study were a selective subgroup of HIV-l-infected individuals and different patterns of mutations may arise in other patient groups.

DNA sequence analysis suggested that the continued evolution of zidovudine-resistant genotypes involves the turnover of virus populations during the course of treatment. The presence of different virus populations has been studied for a number of HIV genes, rev (Pedroza Martins et al., 1991), nef(Delassus et al., 1991), tat (Meyerhans et al., 1989) and env (Shen Pang et al., 1991; Simmonds et al., 1991), but similar studies have not been performed on RT. Work on other HIV genes has indicated that no two loci evolve at the same rate (Pedroza Martins et al., 1991) so care must be taken in comparing conclusions. The limited sequence analysis presented here revealed the presence of distinct virus populations at different time points, with each population being identified by groups of zidovudine resistance mutations and additional silent mutations. This suggests that there was an evolution of HIV species during drug therapy.

Recombination has been identified as a mechanism for generating rapid genetic diversity in retroviruses including HIV (Beemon et al., 1974; Blair, 1977; Hu \& Temin, 1990; Linial \& Blair, 1982). It is possible that recombination events led to the linkage of mutations at codons 41 and 215 . Tentative evidence for this is provided in the patients' isolates studied. In Pt199 strain C199/ $36 / 6$ contains the codon 41 mutation with a silent mutation at codon 34. Recombination of this species between codons 70 to 214 with either strain C199/36/1 or C199/36/2 would result in the species represented by C199/36/8. It is possible that this genotype could arise from PCR-mediated recombination in the 36 week sample (Meyerhans et al., 1990). However, a similar candidate for recombination exists in Pt105 isolates, where recombination between $\mathrm{C} 105 / 24 / 5$ and $\mathrm{C} 105 /$ $24 / 7$ would give rise to the species C105/24/1. This particular species represented $30 \%$ (three) of the clones sequenced and persisted in the 60 week samples as species C105/60/2, indicating that artefactual PCR-mediated recombination is an unlikely explanation.

Taken together, the sequence analysis, growth properties and drug sensitivities derived from the assay of site- directed mutant viruses suggest that virus outgrowth is conferred by the selective advantage mediated primarily by increases in the level of drug resistance. However, care must be taken in extrapolating these results to a clinical setting. Although the pattern of mutation acquisition was consistent between patients, the rate of appearance did vary. This may reflect other in vivo factors that affect the rate of mutation acquisition. Growth in vivo is determined by many factors. In longitudinal studies nonsyncytium-inducing (NSI) isolates have been shown to be present throughout HIV-1 infection, whereas syncytiuminducing (SI) variants generally arise during the course of infection (Koot et al., 1992; Schuitemaker et al., 1990). Subjects harbouring SI HIV isolates have a much more rapid course of disease than those harbouring NSI variants (Tersmette et al., 1989). Recent work has suggested that rapid disease progression during zidovudine treatment is linked to the presence of SI isolates and the faster appearance of the combined 41 and 215 amino acid changes (Boucher et al., 1992b).

The exact amino acid substitutions in the different combinations of mutations are also critical. HIVRTMR $(41,67,70,215 \mathrm{~F}, 219)$ differs from HIVRTMS $(41,67$, $70,215 \mathrm{Y}, 219)$ only at codon 215 , having a Phe rather than Tyr. The combination in HIVRTMR confers a consistent threefold decrease in resistance to zidovudine relative to HIVRTMS. It is interesting to note that of 56 RT genes sequenced from the two patients Pt199 and Pt 105 no evidence of a Phe at codon 215 was obtained. The level of drug resistance of other combinations of mutations with Phe-215 is not known, and the relative frequency of Phe-215 compared to Tyr-215 in patient groups is also unknown. However, it is unlikely that highly resistant viruses that contain a mutation at codon 41 will also contain a Phe at codon 215 . Other mutations in HIV RT have been implicated in zidovudine resistance although none has been introduced into defined genetic backgrounds to prove their contribution (Gingeras et al., 1991; Japour et al., 1991; Sheehy \& Desselberger, 1993). In this study, variation in $\mathrm{RT}$ was also observed resulting in amino acid substitutions. However, it is likely that these changes represent strain variation rather than contributing to zidovudine resistance, for example the mutation Phe-214 to Leu in patient 105 was present before zidovudine therapy, and persisted throughout.

In conclusion, we propose a model for the overall acquisition pattern of the five resistance mutations in asymptomatic patients (Fig. 6). Each new mutant population that becomes dominant has a significant increase in resistance to zidovudine resulting in a selective advantage which enables the virus to replicate and outgrow the previous less resistant variant.

We thank Dr G. Darby, Dr J. M. A. Lange and Dr J. W. Mulder for 
their continued support and interest. We thank Sharon Kemp for providing the site-directed mutant RT RTMU, and Susan Hartman for DNA sequencing work. We also thank Dr Lee Ratner for the clone HXB2, and Dr Jean-François Nicolas for the cell line HT4LacZ-1.

\section{References}

Albert, J., Wahlberg, J., Lundeberg, J., Cox, S., Sandstrom, E., Wahren, B. \& Uhlen, M. (1992). Persistence of azidothymidineresistant human immunodeficiency virus type 1 RNA genotypes in posttreatment sera. Journal of Virology 66, 5627-5630.

Beemon, K., Duesberg, P. \& Vogt, P. (1974). Evidence for crossingover between avian tumor viruses based on analysis of viral RNAs. Proceedings of the National Academy of Sciences, U.S.A. 71, 4254-4258.

BlaIR, D. G. (1977). Genetic recombination between avian leukosis and sarcoma viruses. Experimental variables and the frequencies of recombination. Virology 77, 534-544.

Boucher, C. A. B., Tersmette, M., Lange, J. M. A., Kellam, P., De Goede, R. E. Y., Mulder, J. W., Darby, G., Goudsmit, J. \& LARDER, B. A. (1990). Zidovudine sensitivity of human immunodeficiency virus from high-risk, symptom free individuals during therapy. Lancet 336, 585-589.

Boucher, C. A. B., O'Sullivan, E., Mulder, J. W., Ramautarsing, C., Kellam, P., Darby, G., Lange, J. M. A., Goudsmit, J. \& LARDER, B. A. (1992a). Ordered appearance of zidovudine resistant mutations during treatment of 18 human immunodeficiency virus positive subjects. Journal of Infectious Diseases 165, 105-110.

Boucher, C. A. B., Lange, J. M. A., Miedema, F. F., Weverling, G. J., Koot, M., Mulder, J. W., Goudsmit, J., Kellam, P., Larder, B. A. \& Tersmetre, M. (1992b). HIV-l biological phenotype and the development of zidovudine resistance in relation to disease progression in asymptomatic individuals during treatment. AIDS 6, 1259-1264.

Boucher, C. A. B., Van Leeuwen, R., Kellam, P., Schipper, P., TiJnagel, J., LANGE, J. M. A. \& LARder, B. A. (1993). Effects of discontinuation of zidovudine treatment on the zidovudine sensitivity of human immunodeficiency virus type 1 isolates. Antimicrobial Agents and Chemotherapy 37, 1525-1530.

Chesebro, B. \& Wehrly, K. (1988). Development of a sensitive quantitative focal assay for human immunodeficiency virus infectivity. Journal of Virology 62, 3779-3788.

Delassus, S., Cheyneir, R. \& Wain Hobson, S. (1991). Evolution of human immunodeficiency virus type 1 nef and long terminal repeat sequences over 4 years in vitro and in vivo. Journal of Virology 65 , 225-231.

Firzgibbon, J. E., Howell, R. M., Haberzettl, C. A., Sperber, S. J., Gocke, D. J. \& Dubin, D. T. (1992). Human immunodeficiency virus type 1 pol gene mutations which cause decreased susceptibility to 2'-,3'-dideoxycytidine. Antimicrobial Agents and Chemotherapy 36, 153-157.

Gingeras, T. R., Prodanovich, P., Latimer, T., Guatelli, S. C, RIChMAN, D. D. \& Barringer, K. J. (1991). Use of the selfsustained sequence replication amplification reaction to analyse and detect mutations in zidovudine-resistant human immunodeficiency virus. Journal of Infectious Diseases 164, 1066-1074

Goodenow, M., Huet, T., SAurin, W., Kwok, S., SNinsky, J. \& Wain Honson, S. (1989). HIV-1 isolates are rapidly evolving quasispecies: evidence for viral mixtures and preferred nucleotide substitutions. Joumal of Acquired Immune Deficiency Syndromes 2, 344-352.

Harada, S., Koyanagi, Y. \& Yamamoto, N. (1985). Infection of HTLV-III/LAV in HTLV-I carrying cells MT-2 and MT-4 and application in a plaque assay. Science $\mathbf{2 2 9}, 563-566$.

HU, W. S. \& TEMIN, H. M. (1990). Genetic consequences of packaging two RNA genomes in one retroviral particle: pseudodiploidy and high rate of genetic recombination. Proceedings of the National Academy of Sciences, U.S.A. 87, 1556-1560.

Japour, A. J., Chatis, P. A., Eigenrauch, H. A. \& Crumpacker, C. S. (1991). Detection of human immunodeficiency virus type 1 clinical isolates with reduced sensitivity to zidovudine and dideoxy. inosine by RNA:RNA hybridization. Proceedings of the National Academy of Sciences, U.S.A. 88, 3092-3096.

Kellam, P., Boucher, C. A. B. \& Larder, B. A. (1992). Fifth mutation in human immunodeficiency virus type 1 reverse transcriptase contributes to the development of high-level resistance to zidovudine. Proceedings of the National Academy of Sciences, U.S.A. 89, 1934-1938.

Koot, M., Vos, A. H. V., Keet, R. P. M., De Goede, R. E. Y., Dercksen, M. W., Terpstra, F. G., Coutinho, R. A., Miedema, F. \& TERSMETTE, M. (1992). HIV-1 biological phenotype in long term infected individuals evaluated with an MT-2 cocultivation assay. AIDS 6, 49-54.

land, S., Treloaer, G., McPhee, D., Birch, C., Dohert, R., Cooper, D. \& GUST, I. (1990). Decreased in vitro susceptibility to zidovudine of HIV isolates obtained from patients with AIDS. Journal of Infectious Diseases 161, 326-329.

Land, S., MCGavin, K., BirCH, C. \& LuCas, R. (1991). Reversion from zidovudine resistance to sensitivity on cessation of treatment. Lancet 338, 830-831.

LARDER, B. A. (1992). 3'-Azido-3'-deoxythymidine resistance suppressed by a mutation conferring human immunodeficiency virus type 1 resistance to nonnucleoside reverse transcriptase inhibitors. Antimicrobial Agents and Chemotherapy 36, 2664-2669

LARDER, B. A. \& KeMP, S. D. (1989). Multiple mutations in HIV-1 reverse transcriptase confer high-level resistance to zidovudine (AZT). Science 246. 1155-1158.

Larder, B. A., Darby, G. \& Richman, D. D. (1989), HIV with reduced sensitivity to zidovudine (AZT) isolated during prolonged therapy. Science 243, 1731-1734.

Larder, B. A., Chesebro, B. \& Ruchman, D. D. (1990). Susceptibilities of zidovudine-susceptible and -resistant human immunodeficiency virus isolates to antiviral agents determined by using a quantitative plaque reduction assay. Antimicrobial Agents and Chemotherapy 34, 436-441.

Larder, B. A., Kellam, P. \& KemP, S. D. (1991). Zidovudine resistance predicted by direct detection of mutations in DNA from HIV-infected lymphocytes. AIDS 5, 137-144.

LINIAL, M. \& BLAIR, D. (1982). Genetics of retroviruses. In $R N A$ Tumor Viruses, pp. 649-783. Edited by R. Weiss, N. Teich, H. Varmus \& J. Coffin. New York: Cold Spring Harbor Laboratory.

Meyerhans, A., Cheynier, R., Albert, J., Seth, M., Kwok, S., Sninsky, J., Morfeldt-Manson, L., Asjo, B. \& Wain Hobson, S. (1989). Temporal fluctuations in HIV quasispecies in vivo are not reflected by sequential HIV isolates. Cell $\mathbf{5 8}, 901-910$.

MeYerhans, A., Vartanian, J.-P. \& Wain Hobson, S. (1990). DNA recombination during PCR. Nucleic Acids Research 18, 1687-1691.

Nunberg, J. H., Schleif, W. A., Boots, E. J., O'Brien, J. A., Quintero, J. C., Hoffman, J. M.. Emini, E. A. \& Goldman, M. E. (1991). Viral resistance to human immunodeficiency virus type-1 specific pyridinone reverse transcriptase inhibitors. Joumal of Virology 65, 4887-4892.

Pedroza Martins, L., Chenciner, N., Asjo, B., Meyerhans, A. \& WaIN HoBson, S. (1991). Independent fluctuation of human immunodeficiency virus type $1 \mathrm{rev}$ and gp41 quasispecies in vivo. Journal of Virology 65, 4502-4507.

Perno, C.-F., Yarchoan, R., Balzarini, J., Bergamini, A., Millanese, G., Pauwels, R., De Clerce, E., Rocchi, G. \& Calio, R. (1992). Different pattern of activity of inhibitors of human immunodeficiency virus in lymphocytes and monocyte/macrophages. Antiviral Research 17, 289-304.

Richman, D. D., Grimes, J. M. \& Lagakos, S. W. (1990). Effect of stage of disease and drug dose on zidovudine susceptibilities of isolates of human immunodeficiency virus. Journal of Acquired Immune Deficiency Syndromes 3, 743-746.

Richman, D. D., Shih, C. K., Lowy, I., Rose, J., Prodanovich, P., GofF, S. \& Grifrin, J. (1991 a). Human immunodeficiency virus type 1 mutants resistant to non-nucleoside inhibitors of reverse transcriptase arise in tissue culture. Proceedings of the National Academy of Sciences, U.S.A. 88, 11241-11243.

Richman, D. D., Guatelli, J. C., Grimes, J., Tsiatis. A. \& Gingeras, T. $(1991 b)$. Detection of mutants associated with zidovudine 
resistance in human immunodeficiency virus by use of the polymerase chain reaction. Journal of Infectious Diseases 164, 1075-1081.

Rocancourt, D., Bonnerot, C., Jouin, H., Emerman, M. \& Nicolas, J.-F. (1990). Activation of a $\beta$-galactosidose recombinant provirus: application to titration of human immunodeficiency virus (HIV) and HIV-infected cells. Journal of Virology 64, 2660-2668.

Rooke, R., Tremblay, M., Soudeynes, H., De Stephano, L., Yao, X.-J., Fanning, M., Montaner, J. S. G., O'Shaughnessy, M., Gelman, K., Tsoukas, C., Gill, J., Ruedy, J. \& Wainberg, M. A. (1989). Isolation of drug-resistant variants of HIV-l from patients on long term zidovudine therapy, AIDS 3, 411-415.

Salahuddin, S.Z., Markham, P. D., Wong-Staal, F., Franchini, G., Kalyanaraman, V.S. \& Gallo, R. C. (1983). Restricted expression of human T-cell leukemia-lymphoma virus (HTLV) in transformed human umbilical cord blood lymphocytes. Virology 129, 51-64.

Sanger, F., Nicklen, S. \& Coulson, A. R. (1977). DNA sequencing with chain-terminating inhibitors. Proceedings of the National Academy of Sciences, U.S.A. 74, 5463-5467.

Schuitemaker, H., Kootstra, N. A., De Goede, F, De Wolf, F., Miedema, F. \& Tersmette, M. (1990). Monocytotropic human immunodeficiency virus 1 (HIV-1) variants detectable in all stages of HIV infection are predominantly lacking T-cell line tropism and syncytium-inducing ability in primary T-cell culture. Journal of Virology 65, 356-363.

SchuUrman, R. \& Keulen, W. (1990). Modified protocol for DNA sequence analysis using Sequenase 2.0. Biotechniques 11, 185.

SHEEHY, N. \& DEsselberger, U. (1993). Sequence analysis of reverse transcriptase genes of zidovudine (AZT)-resistant and -sensitive human immunodeficiency virus type 1 strains. Journal of General Virology 74, 223-228.

Shen Pang, H., Akashi, V., O'Brien, W. A. \& Chen, I. S. Y. (1991). HIV-1 env sequence variation in brain tissue of patients with AIDS related neurologic disease. Journal of Acquired Immune Deficiency Syndromes 4, 1082-1092.

Simmonds, P., Zhang, L. Q., McOmish, F., Balfe, P., Ludlam, C. A. \& LEIGH-BRowN, A. J. (1991). Discontinuous sequence change of human immunodeficiency virus (HIV) type 1 env sequences in the plasma viral and lymphocyte associated proviral populations in yivo: implications for models of HIV pathogenesis. Journal of Virology 65 , 6266-6276

St Clatr, M. H., Martin, J. L., Tudor-Williams, G., Bach, M. C., Vayro, C. L., King, D. M., Kellam, P., Kemp, S. D. \& Larder, B. A. (1991). Resistance of ddI and sensitivity to AZT induced by a mutation in HIV-1 reverse transcriptase. Science 253, 1557-1559.

Tersmette, M., Lange, J. M. A., De Goede, R. E. Y., De Wolf, F., Beftink-Schattenkerk, J. K. M., Schellekens, P. T. W. A. Coutinho, R. A., Huisman, J. G., Goudsmit, J. \& Miedema, F. (1989). Association between biological properties of human immunodeficiency virus variants and risk for AIDS and AIDS mortality. Lancet i, 983-985.

Zoller, M. J. \& SMITH, M. (1984). Oligonucleotide-directed mutagenesis: a simple method using two oligonucleotide primers and a single-stranded DNA template. DNA 3, 479-488.

(Received 23 July 1993; Accepted 24 September 1993) 